\title{
Palliative Care Needs in Oncology, Cardiology, and Neurology Clinic Patients in the USA
}

\author{
Nancy Dudley, PhD, MSN ${ }^{1,2}$, Christine S. Ritchie, $M D, M S P H^{7}$, Irena Stijacic-Cenzer, $\mathrm{MA}^{7}$, and \\ Sei J. Lee, $M D^{1,2}$ \\ 'Division of Geriatrics, Department of Medicine, University of California, San Francisco, CA, USA; ${ }^{2}$ Geriatrics, Palliative \& Extended Care, San \\ Francisco Veterans' Affair Medical Center, San Francisco, CA, USA.
}

J Gen Intern Med 34(7):1100-2

DOI: $10.1007 / \mathrm{s} 11606-019-04854-6$

(c) Society of General Internal Medicine 2019

\section{INTRODUCTION}

The Institute of Medicine's 2014 report Dying in America calls for all clinicians to provide symptom-oriented palliative care to patients, especially for the growing population of older adults with advanced illness. ${ }^{1}$ Oncology, cardiology, and neurology professional societies all recommend their members complement usual disease-oriented care with symptomoriented palliative care services for patients with advanced illness. $^{2-4}$ However, the proportion of oncology, cardiology, and neurology patients who have advanced illness and are most likely to benefit from symptom-oriented palliative care is unclear.

\section{METHODS}

We conducted a cross-sectional analysis of the National Ambulatory Medical Care Surveys (NAMCS) (2009-2011), a nationally representative survey of ambulatory care patient visits to non-federal office-based physicians. We used chisquare tests to compare differences across US oncology, neurology, and cardiology clinics among adults 65 years and older with advanced illness. Advanced illness was defined by ICD-9 codes from the NCQA Palliative and End-of-Life Care Physician Performance Measurement Set. ${ }^{5}$ We examined sociodemographic characteristics and prevalence of multimorbidity. Results were adjusted for survey weights and design factors to provide nationally representative estimates. This study was exempted from review by the IRB of the University of California, San Francisco.

\section{RESULTS}

Between 2009 and 2011, 14.0\% of visits among adults 65 and older to US oncology, neurology, and cardiology clinics were by patients with advanced illness (Table 1). The rates of advanced illness visits across clinics were different for neurology $(22.1 \%)$, oncology $(19.2 \%)$, and cardiology $(9.6 \%)$

Published online February 7, 2019 $(p<0.001)$. There were substantial differences in the proportion of advanced illness visits by age and gender. Among adults 65-74, a higher proportion of oncology (20.8\%) visits were advanced illness visits; among adults 75+, a higher proportion of neurology (28.3\%) visits were advanced illness visits. For both men and women, the highest proportion of advanced illness visits occurred in neurology clinics (both $p<0.001$ ).

Other characteristics related to advanced illness visits were socioeconomic factors, insurance, and prevalence of multimorbidity. For Hispanic patients, nearly 1 in 3 neurology $(29.4 \%)$ visits were advanced illness visits. In contrast to nonHispanic Black patients, only 1 in 6 neurology (16.6\%) visits were advanced illness visits. For insurance, Medicaid oncology patients had the highest rates of advanced illness $(41.6 \%$, $95 \%$ CI 25.9, 59.1). This was substantially higher than the rates of advanced illness among Medicare oncology patients $(18.5 \%, 95 \%$ CI 15.9, 21.5), Medicaid neurology patients $(22.5 \%, 95 \%$ CI 9.2, 45.5), and Medicaid cardiology patients $(4.3 \%, 95 \%$ CI 1.3, 13.1). Patients with multimorbidity, defined as three or more chronic conditions, had nearly twice the rate of cardiology advanced illness visits than patients without multimorbidity $(13.1 \%$ vs. $6.1 \% ; p<0.001)$. Rural patients had high rates of oncology advanced illness visits compared to non-rural patients (33.6\% vs. 18.7\%). Although differences are noted in advanced illness visits across oncology, neurology, and cardiology clinics, overall, our results show that advanced illness visits are common to these practices.

\section{DISCUSSION}

With $14.0 \%$ (or 1 in 7 ) of patient visits to oncology, neurology, and cardiology clinics involving individuals with advanced illness, our results suggest that many patients seen in these clinics would benefit from symptomoriented palliative care in conjunction with usual diseaseoriented care. ${ }^{6}$ Specifically, our results suggest that 1 in 5 patients seen in oncology and neurology clinics have advanced illness and would likely benefit from a symptom-oriented palliative approach. One in 10 patients in cardiology clinics have advanced illness and would likely benefit from a palliative approach. In addition, the 
Table 1 Characteristics of Adults Age 65+ Seen in US Medical Specialty Clinics With Advanced Illness, NAMCS 2009-2011 (Weighted $(95 \% \mathrm{CI}))$

\begin{tabular}{|c|c|c|c|c|c|c|c|c|c|c|}
\hline \multirow{2}{*}{$\begin{array}{l}\text { Patient } \\
\text { characteristics }\end{array}$} & \multicolumn{3}{|c|}{ Oncology } & \multicolumn{3}{|l|}{ Neurology } & \multicolumn{3}{|c|}{ Cardiology } & \multirow{2}{*}{$\begin{array}{l}\begin{array}{l}\text { Across } \\
\text { clinics }\end{array} \\
p \text { value }\end{array}$} \\
\hline & $\begin{array}{l}\text { Total } \\
N \\
\text { (in } \\
\mathbf{1 0 0 0 ~ s )}\end{array}$ & $\begin{array}{l}\text { Advanced } \\
\text { illness } \\
(\%, C I)\end{array}$ & $\begin{array}{l}p \\
\text { value }\end{array}$ & $\begin{array}{l}\text { Total } N \\
\text { (in } 1000 \mathrm{~s} \text { ) }\end{array}$ & $\begin{array}{l}\text { Advanced } \\
\text { illness } \\
(\%, \mathrm{CI})\end{array}$ & $p$ value & $\begin{array}{l}\text { Total } \\
N \\
\text { (in } \\
\text { 1000s) }\end{array}$ & $\begin{array}{l}\text { Advanced } \\
\text { illness } \\
(\%, C I)\end{array}$ & $p$ value & \\
\hline Unweighted, no. & 1329 & & & 1602 & & & 3117 & & & \\
\hline Weighted, no. & 20,957 & $\begin{array}{l}19.2 \% \\
(16.7,21.9)\end{array}$ & & 13,572 & $\begin{array}{l}22.1 \% \\
(19.1,25.4)\end{array}$ & & 51,834 & $\begin{array}{l}9.6 \% \\
(8.3,11.2)\end{array}$ & & $<0.001$ \\
\hline $\begin{array}{l}\text { Age } \\
65-74\end{array}$ & 10,933 & $\begin{array}{l}20.8 \% \\
(15.9,26.8)\end{array}$ & 0.43 & 6487 & $\begin{array}{l}15.3 \% \\
(11.4,20.4)\end{array}$ & $<0.001$ & 22,486 & $\begin{array}{l}7.1 \% \\
(5.7,8.9)\end{array}$ & $<0.001$ & $<0.001$ \\
\hline $75+$ & 10,024 & $\begin{array}{l}17.4 \% \\
(13.5,22.2)\end{array}$ & & 7086 & $\begin{array}{l}28.3 \% \\
(24.6,32.2)\end{array}$ & & 29,348 & $\begin{array}{l}11.5 \% \\
(9.9,13.5)\end{array}$ & & $<0.001$ \\
\hline Gender & & & & & & & & & & \\
\hline Female & 11,461 & $\begin{array}{l}16.2 \% \\
(13.0,20.0)\end{array}$ & 0.01 & 7776 & $\begin{array}{l}19.3 \% \\
(16.1,22.9)\end{array}$ & 0.01 & 26,200 & $\begin{array}{l}9.6 \% \\
(8.0,11.5)\end{array}$ & 0.96 & $<0.001$ \\
\hline Male & 9495 & $\begin{array}{l}22.8 \% \\
(19.4,26.5)\end{array}$ & & 5797 & $\begin{array}{l}25.9 \% \\
(21.5,30.8)\end{array}$ & & 25,634 & $\begin{array}{l}9.7 \% \\
(8.0,11.6)\end{array}$ & & $<0.001$ \\
\hline $\begin{array}{l}\text { Race/ethnicity } \\
\text { Non-Hispanic } \\
\text { White }\end{array}$ & 15,432 & $\begin{array}{l}18.9 \% \\
(16.1 .22 .2)\end{array}$ & 0.71 & 10,759 & $\begin{array}{l}22.1 \% \\
(18.9 .25 .7)\end{array}$ & 0.08 & 38,980 & $\begin{array}{l}9.5 \% \\
(8.0,11.3)\end{array}$ & 0.98 & $<0.001$ \\
\hline $\begin{array}{l}\text { Non-Hispanic } \\
\text { Black }\end{array}$ & 1738 & $\begin{array}{l}21.9 \% \\
(11.8,37.0)\end{array}$ & & 982 & $\begin{array}{l}16.6 \% \\
(9.0,28.7)\end{array}$ & & 3935 & $\begin{array}{l}9.9 \% \\
(5.2,17.9)\end{array}$ & & 0.10 \\
\hline Hispanic & 3259 & $\begin{array}{l}20.0 \% \\
(13.2,29.1)\end{array}$ & & 1320 & $\begin{array}{l}29.4 \% \\
(21.9,38.2)\end{array}$ & & 5178 & $\begin{array}{l}9.5 \% \\
(6.6,13.5)\end{array}$ & & $<0.001$ \\
\hline $\begin{array}{l}\text { Non-Hispanic } \\
\text { other } \\
\text { Education } \\
\text { (\%>= bachelor) }\end{array}$ & 527 & $\begin{array}{l}12.5 \% \\
(5.8,25.0)\end{array}$ & & 512 & $\begin{array}{l}13.0 \% \\
(6.6,24.3)\end{array}$ & & 3740 & $\begin{array}{l}10.5 \% \\
(4.8,21.5)\end{array}$ & & 0.84 \\
\hline $\begin{array}{l}\text { Quartile } 1 \\
\text { (less than } 12.84 \% \text { ) }\end{array}$ & 5686 & $\begin{array}{l}24.7 \% \\
(19.2,31.1)\end{array}$ & 0.14 & 3044 & $\begin{array}{l}17.6 \% \\
(13.4,22.7)\end{array}$ & 0.28 & 12,307 & $\begin{array}{l}8.9 \% \\
(6.8,11.4)\end{array}$ & 0.26 & $<0.001$ \\
\hline $\begin{array}{l}\text { Quartile 2 } \\
(12.84-19.66 \%)\end{array}$ & 4782 & $\begin{array}{l}18.1 \% \\
(12.5,25.6)\end{array}$ & & 3223 & $\begin{array}{l}23.9 \% \\
(19.1,29.5)\end{array}$ & & 10,541 & $\begin{array}{l}9.6 \% \\
(6.9,13.2)\end{array}$ & & $<0.001$ \\
\hline $\begin{array}{l}\text { Quartile } 3 \\
(19.67-31.68 \%\end{array}$ & 4815 & $\begin{array}{l}16.2 \% \\
(11.7,22.0)\end{array}$ & & 3013 & $\begin{array}{l}22.2 \% \\
(18.2,26.8)\end{array}$ & & 12,915 & $\begin{array}{l}11.6 \% \\
(9.2,14.5)\end{array}$ & & $<0.001$ \\
\hline $\begin{array}{l}\text { Quartile } 4 \\
\text { (31.69\% or more) } \\
\text { Insurance }\end{array}$ & 4681 & $\begin{array}{l}15.8 \% \\
(10.8,22.4)\end{array}$ & & 3363 & $\begin{array}{l}21.9 \% \\
(16.9,28.0)\end{array}$ & & 14,154 & $\begin{array}{l}8.5 \% \\
(6.6,10.8)\end{array}$ & & $<0.001$ \\
\hline Private & 2873 & $\begin{array}{l}21.9 \% \\
(16.3,28.9)\end{array}$ & 0.01 & 1995 & $\begin{array}{l}23.0 \% \\
(17.0,30.4)\end{array}$ & 0.95 & 7344 & $\begin{array}{l}8.9 \% \\
(5.7,13.6)\end{array}$ & 0.36 & $<0.001$ \\
\hline Medicare & 17,149 & $\begin{array}{l}18.5 \% \\
(15.9,21.5)\end{array}$ & & 10,581 & $\begin{array}{l}22.0 \% \\
(18.7,25.6)\end{array}$ & & 41,540 & $\begin{array}{l}10.1 \% \\
(8.7,11.7)\end{array}$ & & $<0.001$ \\
\hline Medicaid & 379 & $\begin{array}{l}41.6 \% \\
(25.9,59.1)\end{array}$ & & 269 & $\begin{array}{l}22.5 \% \\
(9.2,45.5)\end{array}$ & & 1104 & $\begin{array}{l}4.3 \% \\
(1.3,13.1)\end{array}$ & & $<0.001$ \\
\hline \multicolumn{11}{|l|}{$\begin{array}{l}\text { Visits } 3+ \\
\text { chronic conditions }\end{array}$} \\
\hline No & 14,186 & $\begin{array}{l}17.9 \% \\
(15.2,21.0)\end{array}$ & 0.15 & 10,015 & $\begin{array}{l}22.6 \% \\
(19.0,26.6)\end{array}$ & 0.50 & 25,785 & $\begin{array}{l}6.1 \% \\
(4.8,7.8)\end{array}$ & $<0.001$ & $<0.001$ \\
\hline Yes & 6770 & $\begin{array}{l}21.9 \% \\
(17.3,27.3)\end{array}$ & & 3557 & $\begin{array}{l}20.7 \% \\
(16.5,25.6)\end{array}$ & & 26,049 & $\begin{array}{l}13.1 \% \\
(11.0,15.6)\end{array}$ & & $<0.001$ \\
\hline \multicolumn{11}{|l|}{$\begin{array}{l}\text { Median household } \\
\text { income patient's } \\
\text { zip code }\end{array}$} \\
\hline $\begin{array}{l}\text { Quartile } 1 \\
\text { (below } \$ 32,793 \text { ) }\end{array}$ & 4777 & $\begin{array}{l}21.7 \% \\
(15.9,28.8)\end{array}$ & 0.33 & 2617 & $\begin{array}{l}22.0 \% \\
(16.8,28.3)\end{array}$ & 0.75 & 11,150 & $\begin{array}{l}9.4 \% \\
(7.7,11.5)\end{array}$ & 0.02 & $<0.001$ \\
\hline $\begin{array}{l}\text { Quartile } 2 \\
(\$ 32,794-\$ 40,626)\end{array}$ & 4716 & $\begin{array}{l}21.3 \% \\
(15.7,28.2)\end{array}$ & & 3093 & $\begin{array}{l}19.5 \% \\
(13.9,26.6)\end{array}$ & & 10,569 & $\begin{array}{l}13.3 \% \\
(10.2,17.2)\end{array}$ & & 0.03 \\
\hline $\begin{array}{l}\text { Quartile } 3 \\
(\$ 40,627-\$ 52,387)\end{array}$ & 5866 & $\begin{array}{l}14.8 \% \\
(10.1,21.3)\end{array}$ & & 3634 & $\begin{array}{l}20.9 \% \\
(17.3,25.1)\end{array}$ & & 12,941 & $\begin{array}{l}7.8 \% \\
(5.5,10.9)\end{array}$ & & $<0.001$ \\
\hline $\begin{array}{l}\text { Quartile } 4 \\
\text { (\$52,388 or more) } \\
\text { MSA }\end{array}$ & 4604 & $\begin{array}{l}19.2 \% \\
(14.2,25.3)\end{array}$ & & 3299 & $\begin{array}{l}23.4 \% \\
(17.9,29.9)\end{array}$ & & 15,258 & $\begin{array}{l}8.8 \% \\
(6.9,11.0)\end{array}$ & & $<0.001$ \\
\hline Non-rural & 20,313 & $\begin{array}{l}18.7 \% \\
(16.2,21.5)\end{array}$ & $\begin{array}{c}< \\
0.001\end{array}$ & 12,630 & $\begin{array}{l}21.6 \% \\
(18.5,25.1)\end{array}$ & 0.17 & 48,886 & $\begin{array}{l}9.8 \% \\
(8.4,11.4)\end{array}$ & 0.14 & $<0.001$ \\
\hline Rural & 644 & $\begin{array}{l}33.6 \% \\
(28.6,39.0)\end{array}$ & & 943 & $\begin{array}{l}28.2 \% \\
(19.8,38.6)\end{array}$ & & 2948 & $\begin{array}{l}7.1 \% \\
(4.6,10.7)\end{array}$ & & $<0.001$ \\
\hline
\end{tabular}

*All estimates (except unweighted) reflect survey-weighted proportions accounting for the complex survey design of the National Medical Care Survey

high rates of advanced illness among rural oncology clinics suggest that interventions such as telehealth may allow for the cost-effective provision of needed palliative care services to this underserved population. 
Our findings have important implications. Patients 65 years and older with advanced illness visits are not rare. Although, some patients with advanced illness may need specialized palliative care requiring referral to palliative care trained physicians, many advanced illness patients may benefit from basic palliative care that can be provided by all clinicians. Thus, there is a huge opportunity to recognize/identify those eligible for palliative care earlier in every clinical practice, and increase palliative care education and resources for all providers, including oncologists, neurologists, and cardiologists. Focused educational interventions in basic palliative care may help oncologists, neurologists, and cardiologists provide optimal symptom-oriented care along with disease-oriented care that is aligned with patient values, goals, and preferences.

Corresponding Author: Nancy Dudley, PhD, MSN; Geriatrics, Palliative \& Extended Care, San Francisco Veterans' Affair Medical Center, 4150 Clement Street (181G), San Francisco, CA 94121, USA (e-mail: Nancy.dudley@ucsf.edu).

\section{Compliance with Ethical Standards:}

This study was exempted from review by the IRB of the University of California, San Francisco.
Conflict of Interest: The authors declare that they do not have a conflict of interest.

Publisher's Note: Springer Nature remains neutral with regard to jurisdictional claims in published maps and institutional affiliations.

\section{REFERENCES}

1. National Academy of Medicine. Dying in America: Improving Quality and Honoring Individual Preferences Near the End of Life. Washington, D.C.: The National Academies Press; 2014.

2. Ferrell BR, Temel JS, Temin S, et al. Integration of Palliative Care Into Standard Oncology Care: American Society of Clinical Oncology Clinical Practice Guideline Update. Journal of clinical oncology: official journal of the American Society of Clinical Oncology. 2017; 35(1): 96-112

3. Braun LT, Grady KL, Kutner JS, et al. American Heart Association Advocacy Coordinating Committee. Palliative care and cardiovascular disease and stroke: a policy statement from the American Heart Association/American Stroke Association. Circulation. 2016;134: e198e225

4. Oliver DJ, Borasio G, Caraceni A, et al. A consensus review on the development of palliative care for patients with chronic and progressive neurological disease. Eur J Neurol. 2016; 23(1):30-38

5. National Committee for Quality Assurance (NCQA). Palliative and End of Life Care: Physician Performance Measurement Set. American Medical Association and National Committee for Quality Assurance; 2008.

6. Kelley AS, Morrison RS. Palliative Care for the Seriously Ill. The New England Journal of Medicine. 2015; 373(8):747-755. 